\title{
Comparison theorems between the preconditioned Gauss-Seidel method and the AOR method for M-matrices
}

\author{
Zhang Shi-guang ${ }^{1, a}$, Zhou Ting ${ }^{2, b}$ \\ ${ }^{1}$ College of Computer and Information Engineering, Henan Normal University, 453007 \\ ${ }^{2}$ The state-owned assets management office, Henan Normal University, 453007 \\ asg201106@163.com, bhouting7606@163.com
}

\begin{abstract}
Keywords: Preconditioned Gauss-Seidel iterative method; AOR iterative method; convergence . Abstract. In this paper, we study a preconditioned Gauss-Seidel iterative method with the preconditioner which proposed in [1] for solving a linear system whose coefficient matrix is a M-matrix. Some corresponding comparison results between the preconditioned Gauss-Seidel iterative method and the basic AOR iterative method are obtained. Finally, a numerical example is given to illustrate our results.
\end{abstract}

\section{Introduction}

Consider the following linear system

$$
A x=b,
$$

where $A$ is an $n \times n$ nonsingular matrix, $x$ and $b$ are $n$-dimensional vectors. If $A$ is split into

$$
A=M-N \text {, }
$$

where $M$ is nonsingular, then the basic iterative method for solving the linear system (1) can be expressed in the form

$$
x^{(k+1)}=M^{-1} N x^{(k)}+M^{-1} b, k=0,1, \ldots
$$

Without loss of generality, let $A=I-L-U$, where $I$ is the identity matrix, $-L$ and $-U$ are strictly lower and upper triangular matrices of $A$, respectively. Then the iteration matrix of the classical AOR iterative method (cf. [2]) for solving the linear system (1) is

$$
T_{r, \omega}=(I-r L)^{-1}[(1-\omega) I+(\omega-r) L+\omega U],
$$

where $\omega$ and $r$ are real number with $\omega \neq 0$.

The iteration matrix of the classical SOR iterative method for solving the linear system (1) is(Note that for $\omega=r$, we get the SOR iterative method.)

$$
T_{\omega}=(I-\omega L)^{-1}[(1-\omega) I+\omega U]
$$

Then the iteration matrix of the classical Gauss-Seidel iterative method for solving the linear system (1) is(Note that for $\omega=r=1$, we get the Gauss-Seidel iterative method.)

$$
T_{G S}=(I-L)^{-1} U
$$

The spectral radius of the iterative matrix is decisive for the convergence and stability of the method, and the smaller it is, the faster the method converges when the spectral radius is smaller than 1. The effective method to decrease the spectral radius is to precondition the linear system, thus we now transform the original system (1) into the preconditioned form

$$
P A x=P b,
$$

where $P \in R^{n \times n}$ is a nonsingular matrix. The corresponding basic iterative method is given in general by

$$
x^{(k+1)}=M_{P}^{-1} N_{P} x^{(k)}+M_{P}^{-1} P b, k=0,1, \ldots,
$$

where $P A=M_{P}-N_{P}$ is a splitting of $P A$ and $M_{P}$ is nonsingular.

In [1], the authors presented a preconditioned AOR method for consistent systems by using the preconditioner $P_{m}^{\alpha}=I+S_{m}{ }^{\alpha}$, where 


$$
S_{m}^{\alpha}=\left[\begin{array}{ccccc}
0 & \multicolumn{5}{c}{-\alpha_{1} a_{1, k_{1}}} \\
0 & 0 & -\alpha_{2} a_{2, k_{2}} & & \\
\mathrm{M} & \mathrm{M} & \mathrm{O} & & \\
0 & 0 & \mathrm{~L} & \mathrm{O} & -\alpha_{n-1} a_{n-1, k_{n-1}} \\
0 & 0 & 0 & \mathrm{~L} & 0
\end{array}\right],
$$

and $\alpha_{i}$ is a parameter, $i=1,2, \mathrm{~K}, n-1, k_{i}=\min j \in\left\{j\left|\max _{j(>i)}\right| a_{i j} \mid, i<n\right\}$.

In this paper, we prove theoretically that if $A$ is a nonsingular $M$-matrix, the spectral radius of precond-itioned Gauss-Seidel iterative method by using the preconditioner is faster than that of AOR method and the SOR iterative method and the Gauss-Seidel iterative method.

\section{Preliminaries}

For convenience, we shall now briefly explain some of the terminologies used in the paper. For $A=\left(a_{i j}\right), \quad B=\left(b_{i j}\right) \in R^{n \times n}$ we write $A \geq B$ if $a_{i j} \geq b_{i j}$ holds for $i, j=1,2, \mathrm{~K}, n$.Calling $A$ nonnegative if $A \geq 0 .\left(a_{i j} \geq 0 ; i, j=1,2, \mathrm{~K}, n\right)$. For a square matrix $A, \rho(A)$ denotes the spectral radius of $A$.

Definition2.1 A matrix $A=\left(a_{i j}\right)$ is called a $Z$-matrix if $a_{i j} \leq 0$ for $i \neq j, Z$-matrix is a nonsingular $M$-matrix, if $A$ is nonsingular and $A^{-1} \geq 0$.

Definition2.2 Let $A$ be a real matrix. The representation $A=M-N$ is called a splitting of $A$ if $M$ is a nonsingular matrix. The splitting is called

(a) regular if $M^{-1} \geq 0, N \geq 0$;

(b) weak regular if $M^{-1} \geq 0, M^{-1} N \geq 0$.

Lemma $2.3^{[4]}$ Let $A$ be a Z-matrix. Then the following statements are equivalent:

(a) $A$ is a nonsingular $M$-matrix.

(b) All principal sub-matrices of $A$ are non-singular $M$-matrices.

(c) All principal minors are positive.

Lemma $2.4^{[5]}$ Let $A^{-1} \geq 0$ and $A=M 0-N^{9}=M-N$ be two weak regular splittings of $A$, If $M^{\sigma^{1}} \leq M^{-1}$ and $N o 0$, then $\rho\left(M \sigma^{1} N \rho \geq \rho\left(M^{-1} N\right)\right.$.

Lemma $2.5^{[6]}$ Let $A$ be a nonsingular $M$-matrix. Then $\rho\left(T_{\omega, r}\right)<1$.

\section{Result and proof}

Throughout this paper, we always assume that $a_{i k_{i}} \neq 0, i=1,2, \mathrm{~K}, n-1$. Let $A=I-L-U$, where $I$ is the identity matrix, $-L$ and $-U$ are strictly lower and upper triangular parts of $A$, respectively. We denote

$$
A_{m}^{\alpha}=\left(I+S_{m}{ }^{\alpha}\right) A=\left(I+S_{m}{ }^{\alpha}\right)(I-L-U)=I-L-U+S_{m}{ }^{\alpha}-S_{m}{ }^{\alpha} L-S_{m}{ }^{\alpha} U .
$$

Then the iterative matrix of the preconditioned Gauss-Seidel iterative method is

$$
T_{m}^{\alpha}=\left(I-L-D_{\alpha}^{\prime}-L_{\alpha}^{\prime} F_{\alpha}\right)^{-1}\left(U-S_{m}{ }^{\alpha}+S_{m}^{\alpha} U+F_{\alpha}^{\prime}\right),
$$

where $D_{\alpha}^{\prime}, E_{\alpha}^{\prime}$ and $F_{\alpha}^{\prime}$ are the diagonal, strictly lower and upper triangular parts of $S_{m}{ }^{\alpha} L$, respectively.

Theorem 3.1. Let $A=\left(a_{i j}\right) \in R^{n \times n}$ be a nonsingular $M$-matrix. Assume that $0 \leq r \leq \omega \leq 1, \omega \neq 0$. Let $T_{r, \omega}$ and $T_{m}{ }^{\alpha}$ be defined by (2) and (7), for $\alpha_{i} \in[0,1], i=1,2, \mathrm{~K}, n-1, \rho\left(T_{m}{ }^{\alpha}\right) \leq \rho\left(T_{\omega, r}\right)<1$.

Proof. Let

$$
E_{\alpha}=I-L-D_{\alpha}^{\prime}-L_{\alpha}^{\prime}, F_{\alpha}=U-S_{m}^{\alpha}+S_{m}^{\alpha} U+F_{\alpha}^{\prime},
$$


As $U \geq S_{m}{ }^{\alpha}$, it is clear that $F_{\alpha} \geq 0$. We know that the diagonal elements of $E_{\alpha}$ are $1-\alpha_{i} a_{i, k_{i}} a_{k_{i}, i}$, $i \neq n$ and $a_{n n}=1$. Since $A$ is a nonsingular $M$-matrix, it is clear that all principal minors of $A$ are positive by Lemma 2.3 . Since all the diagonal entries of $A$, we have $1-a_{i, k_{i}} a_{k_{i}, i}>0$. As $\alpha_{i} \in[0,1], i=1,2, \mathrm{~K}, n-1$, we have $1-\alpha_{i} a_{i, k_{i}} a_{k_{i}, i}>0$. This implies that all the diagonal entries of $E_{\alpha}$ are positive, so we know that $E_{\alpha}$ is a nonsingular $M$-matrix by Lemma 2.3. Thus we have $E_{\alpha}{ }^{-1} \geq 0$ by Definition 2.1 and then $E_{\alpha}{ }^{-1} F_{\alpha} \geq 0$. Notice that $I+S_{m}{ }^{\alpha}$ is nonsingular and let

$$
M_{\alpha}=\left(I+S_{m}{ }^{\alpha}\right)^{-1} E_{\alpha}, N_{\alpha}=\left(I+S_{m}{ }^{\alpha}\right)^{-1} F_{\alpha},
$$

then

Since

$$
M_{\alpha}{ }^{-1}=E_{\alpha}^{-1}\left(I+S_{m}^{\alpha}\right) \geq 0, M_{\alpha}^{-1} N_{\alpha}=E_{\alpha}^{-1} F_{\alpha} \geq 0 .
$$

we obtain

$$
A_{m}^{\alpha}=\left(I+S_{m}^{\alpha}\right) A=E_{\alpha}-F_{\alpha},
$$

$$
A=M_{\alpha}-N_{\alpha},
$$

and $A=M_{\alpha}-N_{\alpha}$ is a weak regular splitting of $A$ by Definition 2.2.

As

$$
A=\frac{1}{\omega}(I-r L)-\frac{1}{\omega}[(1-\omega) I+(\omega-r) L+\omega U],
$$

let

$$
M_{\omega, r}=\frac{1}{\omega}(I-r L), N_{\omega, r}=\frac{1}{\omega}[(1-\omega) I+(\omega-r) L+\omega U],
$$

since $0 \leq r \leq \omega \leq 1, \omega \neq 0$, we have

$$
M_{\omega, r} \geq 0, N_{\omega, r} \geq 0 .
$$

So $A=M_{\omega, r}-N_{\omega, r}=M_{\alpha}-N_{\alpha}$ be two weak splitting of $A$ and $N_{\omega, r} \geq 0$.

By $0 \leq r \leq \omega \leq 1, \omega \neq 0$, we obtain

$$
\frac{1}{\omega}(I-r L)=\frac{1}{\omega} I-\frac{r}{\omega} L \geq I-L \geq I-L-D_{\alpha}^{\prime}-L_{\alpha}^{\prime}=E_{\alpha},
$$

it is clear that

$$
M_{\omega, r} \geq E_{\alpha}
$$

and therefore

$$
M_{\omega, r}^{-1} \leq E_{\alpha}^{-1} \leq E_{\alpha}^{-1}\left(I+S_{m}^{\alpha}\right)=M_{\alpha}{ }^{-1} .
$$

So by Lemma 2.4, we have

$$
\rho\left(T_{m}^{\alpha}\right) \leq \rho\left(T_{\omega, r}\right) .
$$

Thus from Lemma 2.5, we know $\rho\left(T_{\omega, r}\right)<1$, so we obtain

$$
\rho\left(T_{m}^{\alpha}\right) \leq \rho\left(T_{\omega, r}\right)<1 .
$$

Corollary3.2. Let $A=\left(a_{i j}\right) \in R^{n \times n}$ be a nonsingular $M$-matrix. Assume that $0 \leq r \leq \omega \leq 1, \omega \neq 0$. Let $T_{\omega}$ and $T_{m}{ }^{\alpha}$ be defined by (3) and (7), for $\alpha_{i} \in[0,1], i=1,2, \mathrm{~K}, n-1, \rho\left(T_{m}{ }^{\alpha}\right) \leq \rho\left(T_{\omega}\right)<1$.

Proof. We can have the conclusion immediately by let $\omega=r$ in Theorem 3.1.

Corollary3.3. Let $A=\left(a_{i j}\right) \in R^{n \times n}$ be a nonsingular $M$-matrix. Assume that $0 \leq r \leq \omega \leq 1, \omega \neq 0$.

Let $T_{G S}$ and $T_{m}{ }^{\alpha}$ be defined by (4) and (7), for $\alpha_{i} \in[0,1], i=1,2, \mathrm{~K}, n-1, \rho\left(T_{m}{ }^{\alpha}\right) \leq \rho\left(T_{G S}\right)<1$.

Proof. We can have the conclusion immediately by let $\omega=r=1$ in Theorem 3.1. 


\section{Numerical example}

In this section, we give a numerical example to illustrate the comparison theorem of the preconditioned Gauss-Seidel iterative method and the AOR iterative method for solving the linear system (1), where $A$ is an nonsingular $M$ - matrix.

If the coefficient matrix $A$ of (1) is given by

$$
A=\left[\begin{array}{ccccc}
1.000 & -0.097 & -0.103 & -0.271 & -0.268 \\
-0.223 & 1.000 & -0.324 & -0.165 & -0.289 \\
-0.002 & -0.354 & 1.000 & -0.390 & -0.642 \\
-0.007 & -0.291 & -0.393 & 1.000 & -0.106 \\
-0.257 & -0.181 & -0.234 & -0.177 & 1.000
\end{array}\right],
$$

and the parameters $\omega, r$ and $\alpha_{i}, i=1,2, \mathrm{~K}, n-1$ according to the conditions imposed in Theorem 3.1. For the AOR iterative method, with $\omega=0.8$ and $r=0.7$, then $\rho\left(T_{\omega, r}\right)=0.9704$.If we take $\alpha_{1}=0.213$, $\alpha_{2}=0.655, \alpha_{3}=0.964$ and $\alpha_{4}=0.727$, then we have $\rho\left(T_{m}{ }^{\alpha}\right)=0.9308<\rho\left(T_{\omega, r}\right)=0.9704<1$.

From the above numerical results, we obtain the results are in concord with Theorem 3.1.

\section{Acknowledgements}

This work is supported by Ph.D. Research Startup Foundation of Henan Normal University (Nos.qd15129), and Key Project of Science and Technology Department of Henan Province (Nos.17A520038). Please acknowledge collaborators or anyone who has helped with the paper at the end of the text.

\section{References}

[1] Ting Zhou, Shiguang-Zhang, Wenbin-Guo. Improves of preconditioned AOR iterative method for M-matrices,Proceedings of Ninth International Conference on Matrix Theory and its Applications Shanghai, China, July 1822, 2010, pp. 222-225.

[2] A. Hadjimos, Accelerated overrelaxation method, Math. Comp, 32, 1978, pp.149-157.

[3] Varga R S, Matrix Iterative Analysis, Prentice Hall, 1962.

[4] A. Berman, R.J.Plemmons, Nonnegative Matrices in the Mathematical Sciences, SIAM, Philadelphia, PA, 1994.

[5] L. Elsner, Comparisons of weak regular splittings and multisplitting methods, Numer. Math., 56, 1989, pp. 283-289.

[6] Yao-Tang Li, Shun-feng Yang, A multi-parameters preconditioned AOR iterative method for linear systems, Applied Mathematics and Computation, 206, 2008, pp. 465-473. 